\title{
TESTICULAR MICROLITHIASIS
}

\section{Amarawardena $\mathrm{WKMG}^{1}$, Siyambalapitiya $\mathrm{S}^{1}$}

\author{
${ }^{1}$ Diabetes and Endocrine unit, North Colombo Teaching Hospital, Ragama
}

\begin{abstract}
Testicular microlithiasis (TM) is a relatively a common condition of unknown etiology. It is usually benign and asymptomatic. However, these patients can present with testicular pain, fertility problems and the association with testicular cancer is of concern. This is seen in males of different age groups usually as bilateral lesions and the diagnosis is usually made with testicular ultrasonogrpahy. There is no consensus regarding the value of early diagnosis, the probability of developing invasive carcinoma and the necessity for long term follow-up. Majority recommend self-examination of testes as the only regular follow up for this condition. Here we describe a patient with testicular microlithiasis who presented with testicular pain and delayed puberty.
\end{abstract}

\section{INTRODUCTION}

Testicular microlithiasis (TM) is a condition of unknown etiology, which results with the formation of intra-tubular calcifications. It is usually asymptomatic. However, a reasonable percentage of patients experience testicular pain with this condition (1). There is no uniform consensus regarding the management, need for evaluation and method of follow-up of these patients. We report a 16 year old boy, who presented with episodic testicular pain and constitutional delay in growth and puberty with testicular microlithiasis on ultra sound scan of the testes.

\section{CASE REPORT}

16 year old boy presented with three episodes of acute onset testicular pain during the last six months. Each episode lasted for about 12 to 18 hours and completely resolved without any intervention. Episodes were not associated fever or other symptoms of acute inflammation and he denied any history of trauma to genital area. However, he gave a history of mumps at the age of 8 . His antenatal and birth history were unremarkable. He achieved milestones appropriately. At the age of 14 , his parents have noticed that he was short compared to his peers and he had poorly developed secondary sexual characteristics. He did not have galactorrhea, postural dizziness or symptoms of hypothyroidism to suggest other pituitary hormone deficiencies. His testes were $8 \mathrm{ml}$ in size with a phallus and pubic hair of tanner 2 stage. He did not have morphological features of Down's syndrome, Pseudoxanthoma elasticum, Kleinfelter's syndrome, Carney's syndrome.

He was investigated for testicular pain with an ultra sound scan of the testes, which showed bilateral testes within the scrotum with normal echo texture and vascularity.
Testicular sizes were $2.5 \mathrm{~cm} \times 1.5 \mathrm{~cm}$ (right side) and $2.0 \mathrm{~cm} \times 1.3 \mathrm{~cm}$ (left side). Epididymis appeared normal and there was no hydrocele or vericocele. There were microclacifications of more than 20 per single view, which were uniformly distributed in both testes (Figure 1). He was also investigated for delayed puberty and short stature. His thyroid functions and serum prolactin were normal. $\mathrm{LH}$ was $0.342 \mathrm{IU} / \mathrm{L}, \mathrm{FSH}$ was $2.66 \mathrm{IU} / \mathrm{L}$ and total serum testosterone was $0.83 \mathrm{nmol} / \mathrm{L}$. He had delayed bone age. On direct questioning parents revealed a family history of delayed puberty. He was started on puberty induction with intramuscular testosterone enanthate $50 \mathrm{mg}$ monthly. After three months of treatments, he progressed through puberty and his testicular volume increased up to $20 \mathrm{ml}$ and phallus tanner staging of $\mathrm{T} 5$. He gained significant height to achieve his mid parental height.

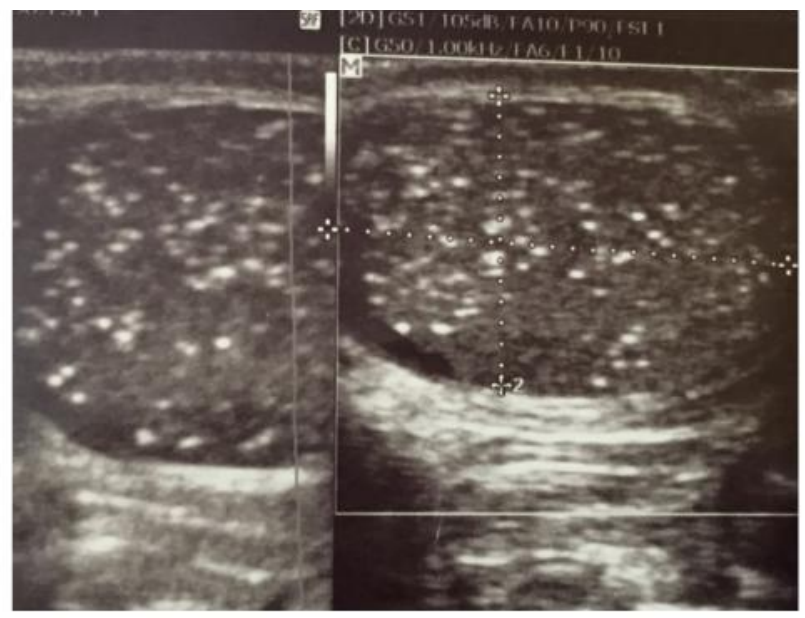

Figure 1: Snow storm appearance of testicular microlithiasis on ultrasound 


\section{DISCUSSION}

Testicular microlithiasis (TM) is a radiological diagnosis and often detected incidentally when scrotal ultrasonogram is done for various indications (1). In a prospective study involving healthy male volunteers (17-42 years of age) using screening scrotal ultrasound scan, a prevalence of $1.1-2.4 \%(1,2)$. It is often seen in males of different age groups, from childhood to old age. However, it is rare in prepubertal boys and in older men more than 60 years of age.

Microliths (calcospherites), are spherical, elongated or ovoid in shape and are eosinophilic. Under the light and electron microscopy, microliths consist two zones: a central calcified zone and a multilayered envelope stratified collagen fiber. Ultrasonically, these are often multiple, uniform, small, echogenic polytopic intratubular calcifications without acoustic shadows. The diagnosis can be made when more than five calcifications are detected.

Although there are occasional cases of unilateral testicular microlithaisis, this condition is usually bilateral in distribution (3). This condition is asymptomatic in majority of patients. However, a reasonable percentage patients experience testicular pain along with this condition $(1,4)$. The pain is thought to be due to the distension of seminiferous tubules. Our patient presented with three episodes of acute onset testicular pain within a period of six months without any ultrasound evidence of inflammation or testicular torsion.

TM is associated with infertility, hypogonadism, testicular infactions, cryptorchidism, male pseudohermaphroditism, varicocele, hydrocele, torsion of testis and its appendages, pseudoxanthoma elasticum, Down's syndrome, Kleinfelter's syndrome, Carney's syndrome, Neurofibromatosis, Pulmonary alveolar microlithiasis, Calcification of sympathetic nervous system and brain, Non-Hodgkin's lymphoma and AIDS (5). In our patient, none of these associations were identified in the history, examination and with the investigations. However, his fertility is yet to be evaluated.

The Leydig cells are not typically affected by testicular microlithiasis (6) However, the majority of the involved seminiferous tubules often has abnormal spermatogonia and reduced luminal diameters (7). Spermatogenesis may be halted at the first order spermatocyte while some patients presented with normal spermatogenesis (7). Relationship between Testicular microlithiasis and infertility is believed to be due to obstruction of semineferous tubules, formed by sloughing of degenerative tubular epithelium.

There are clear guidelines as to how to diagnose testicular microlithiasis ultrasonically and it is graded to minimal, mild moderate and severe (8) (Table 1, 2). According to the ultrasound grading, our patient has severe testicular microlithiasis.
Table 1: Ultrasound criteria for diagnosis of testicular microlithiasis

1. Greater than five calcifications per image field

2. Calcifications less than $2 \mathrm{~mm}$ in diameter

3. Diffuse in nature

4. No acoustic shadowing

5. No loss of testicular shape or volume

Table 2: Severity of the microlithiasis is determined by the number of microliths seen in any single view
1. Minimal / mild (Grade 1)
5-10 microliths
2. Moderate (Grade II)
10-20 microliths
3. Severe (Grade III)
$>20$ microliths

When limited TM is seen in the ultrasound, it should be differentiated from various other conditions causing calcifications, which includes inflammatory (Orchitis, tuberculosis, sarcoidosis, focal scars and post-inflammatory granulomas), vascular (arteritis, chronic infarction), postoperative (post-orchiopexy, sperm granulomas), neoplastic ("burned out" tumors, calcified sertoli cell tumors following radiotherapy and chemotherapy (9). However, these are large, coarse and inhormogenous calcifications that could be easily differentiated from benign testicular microlithiasis.

There is a suggestion that testicular microlithiasis could be associated with male infertility. However, the exact mechanism and the relationship between TM and infertility is unclear. The reported frequency of TM in patients with infertility or undescended testis ranges from $7-39 \%(9,10)$. Obstruction of the seminiferous tubules formed by the slouging degeneratieve tubular epithelium has beed suggested as the underlying cause of TM (9). $30-40 \%$ of the seminiferous tubules get obstructed with intratesticular concretions in these patients, which probably could lead to problems in fertility in these patients.

The association between TM and testicular carcinoma in situ (CIS) has been well documented. In a retrospective analysis of testicular specimens with CIS but without tumors demonstrated TM in $39 \%$ of the specimens compared to $2.1 \%$ in a control group $(P<0.001)(11)$. On the other hand, the results of some prospective studies on asymptomatic men with TM failed to demonstrate the development of interval malignancy during follow up of these patients $(12$, 13). 
At present there is no consensus on the necessity, interval and duration of follow-up and the diagnostic modality to be used. The value of early diagnosis of CIS is still also debatable due to protracted natural history, prolonged follow-up and unknown probability of developing invasive carcinoma. Diagnosis of TM and leaving the patients with the anxiety of subfertility, the potential for cancer and burden of prolonged follow-up of countless years should also be thought of. Therefore, TM can be followed up with regular self-examination of testes. Further evaluation and biopsy is indicated only for the high-risk group (cryptorchidism, infertility, testicular atrophy and contralateral testicular cancer), if any change is noted by the patient and serum markers, provided there is intent to treat (14). Therefore, our patient was advised only on regular self-examination of testes.

\section{CONCLUSION}

TM is usually a benign asymptomatic condition that is prevalent in male population. However, it can present with testicular pain in reasonable percentage of males. The association with male infertility and testicular cancer are of concern for this uncommon lesion of unclear etiology. However, there is no uniform consensus regarding the need for evaluation and method of follow-up of these patients. Therefore, if TM is found in a patient it seems prudent that patients should be educated about the importance of testicular self-examination and encouraged to regular physical examination given the financial constraints in developing countries.

\section{REFERENCES}

1 Dagash $\mathrm{H}^{1}$, Mackinnon EA. Testicular microlithiasis: what does it mean clinically? British Journal of Urology international. 2007; 99(1): 157-160.

2. Janzen DL, Mathieson JR, Marsh JL, Cooperberg PL, delRio P, Golding RH et al. Testicular microlithiasis: Sonographic and clinical features. AJR American Journal of Roentgenol. 1992; 158: 1057-1060.

3. Serter S, Gumus B, Unlu M, Tuncyurek O, Tarhan S, Ayyildiz V et al. Prevalence of testicular microlithiasis in an asymptomatic population. Scandinavian Journal of Urology and Nephrology. 2006; 40: 212-214.

4. Backus ML, Mack LA, Middleton WD, King BF, Winter TC 3rd, True LD. Testicular microlithiasis: Imaging appearance and pathologic correlation. Radiology. 1994; 192: 781-785.

5. Duchek M, Bergh A, Oberg L. Painful testicular lithiasis. Scandinavian Journal of Urology and Nephrology. Supplmentum. 1991; 138: 231-233.

6. Shenykin YR, Goldstein M. Testicular microlithiasis. American Urological Association Update series. 1999; 18 : 106-110.

7. Schantz A, Milstein R. Testicular microlithiasis with sterility. Fertility and Sterility. 1976; 27: 801-805.

8. Weinberg AG, Currarino G, Stone IC Jr. Testicular microlithiasis. Archives of Pathology. 1973; 95: 312-314.

9. Bennett HF, Overdeck DL, Middleton WD. Sonographic follow-up of patients with testicular microlithiasis. Radiology. 1995; 197: 365.
10. Nistal M, Paniaqua R, Diez-Pardo JA. Testicular microlithiais in 2 children with bilateral cryptorchidism. Journal of Urology. 1979; 121: 535537.

11. Vegni-Talluri M, Bigliardi E, Vanni MG, Tota G. Testicular microliths: Their origin and structure. Journal of Urology. 1980; 124: 105-107.

12. Kang JL, Rajpert-De Meyts E, Giwercman A. The association of testicular carcinoma in situ with intratubular microcalcifications. Journal of Urology and Pathology. 1994; 2: 235-242.

13. Janzen DL, Mathieson JR, Marsh JL, Cooperberg PL, delRio P, Golding RH et al. Testicular microlithiasis: Sonographic and clinical features. AJR American Journal of Roentgenol. 1992; 158: $1057-$ 1060.

14. Bennett HF, Overdeck DL, Middleton WD. Sonographic follow-up of patients with testicular microlithiasis. Radiology. 1995; 197: 365.

15. Shanmugasundaram R, Chandra Singh J, Nitin K. Indian Journal of Urology. 2007; 23(3): 234-239. 